\title{
Human allograft acceptance is associated with immune regulation
}

\author{
A.M. VanBuskirk, ${ }^{1}$ W.J. Burlingham,${ }^{2}$ E. Jankowska-Gan, ${ }^{2}$ T. Chin, ${ }^{2}$ S. Kusaka, ${ }^{2}$ \\ F. Geissler, ${ }^{2}$ R.P. Pelletier, ${ }^{1}$ and C.G. Orosz ${ }^{1,3}$ \\ ${ }^{1}$ Department of Surgery, Ohio State University, Columbus, Ohio, USA \\ ${ }^{2}$ Department of Surgery, University of Wisconsin, Madison, Wisconsin, USA \\ ${ }^{3}$ Department of Pathology, Ohio State University, Columbus, Ohio, USA \\ Address correspondence to: Anne M. VanBuskirk, Department of Surgery, Transplantation Division, \\ N937 Doan Hall, 410 West 10th Avenue, Columbus, Ohio 43210, USA. \\ Phone: (614) 293-4087; Fax: (614) 293-8287; E-mail: vanbuskirk.1@osu.edu.
}

A.M. VanBuskirk and W.J. Burlingham contributed equally to this work.

Received for publication December 15, 1999, and accepted in revised form May 16, 2000.

The ultimate goal of transplantation is drug-free allograft acceptance, which is rarely encountered in transplant recipients. Using a novel human-to-mouse "trans vivo" delayed-type hypersensitivity assay, we assessed donor-reactive cell-mediated immune responses in kidney and liver transplant patients, four of whom discontinued all immunosuppression. One of these subjects (J.B.) rejected his graft after 7 years of stable function, while the others (D.S., R.D., M.L.) continue to have excellent graft function 5, 28, and 4 years after the cessation of immunosuppression. PBMCs from J.B. exhibited strong responses to both donor and recall antigens whereas PBMCs from patients D.S., R.D., and M.L. responded strongly to recall, but not donor, antigens. Furthermore, when donor and recall antigens were colocalized, the recall response in these three patients was inhibited. This donor antigen-linked nonresponsiveness was observed in four other patients who are still maintained on immunosuppression. The weakness of donor-reactive DTH responses in these patients is due to donor alloantigen-triggered regulation that relies on either TGF- $\beta$ or IL-10. In D.S., regulation is triggered by a single donor HLA Class I antigen, either in membrane-bound or soluble form. This demonstrates that allograft acceptance in humans is associated with an immune regulation pattern, which may be useful in the diagnosis and/or monitoring of transplant patients for allograft acceptance.

J. Clin. Invest. 106:145-155 (2000).

\section{Introduction}

Transplantation has become an accepted treatment for organ failure for many patients. Nonetheless, one major drawback to this treatment is the need for continuous, indefinite immunosuppression to prevent acute graft rejection. Immunosuppressive therapies have improved dramatically, but the drugs are expensive and associated with undesirable side effects. When patients discontinue immunosuppression, the vast majority of patients rapidly lose graft function. However, a minority of such patients retains graft function (1-4). For the purposes of this study, such long-term drug-free graft acceptance is referred to as "clinical allograft tolerance." Studying the few tolerant patients who have discontinued immunosuppression, yet continue to have excellent graft function, may provide insights into the mechanisms of clinical allograft tolerance.

We have identified previously a specific pattern of immune responses that are associated with allograft acceptance in murine cardiac allograft recipients. These allograft-acceptor animals fail to exhibit donor-reactive delayed-type hypersensitivity (DTH) responses, although they frequently develop donor-reactive alloantibodies (5). We have demonstrated that this absence of donor-reactive DTH is not due to an absence of allosensitization, but to the development of an immune mechanism that actively inhibits antidonor DTH responses. This active regulation exhibits the characteristic of donor antigen-linked DTH nonresponsiveness, or bystander suppression. For example, splenocytes from tetanus toxoid-sensitized (TT-sensitized) allograft acceptor mice, when placed subcutaneously in the ear or footpad of a syngeneic naive mouse, mediate strong DTH responses when challenged with TT, but not with donor alloantigens. When challenged with both TT and donor antigens, the TT-reactive DTH response is lost, demonstrating the dominant negative effect of the response to donor alloantigens (5). This active downregulation is at least partially dependent upon the local activity of TGF- $\beta$ and/or IL-10 (6).

Whereas experimental models of allograft acceptance have been well studied for over 15 years, little of this information has been translated to human clinical transplantation. Indeed, the emphasis in clinical transplantation has recently shifted from the avoidance of acute rejection to an interest in chronic rejection, and most transplant clinicians have yet to consider seriously clinical allograft tolerance. Virtually all of the information 
in the latter area remains anecdotal. One of the underlying difficulties is the lack of simple, rapid, and informative methods with which to assess donor-reactive cellmediated immunity in transplant patients $(7,8)$.

One alternative possibility, traditional skin testing to measure donor-reactive DTH responses in vivo, is potentially very valuable, but not feasible in transplant patients because of the possibility of sensitizing the patients to donor antigens. To avoid this problem, we developed a "trans vivo" DTH assay in which human PBMCs are injected, along with appropriate antigen, into the footpads or pinnae of naive mice. If the PBMC donor has been sensitized previously to the antigen, an antigen-specific, DTH-like swelling develops within 24 hours. This response requires prior antigen sensitization of the PBMC donor, is antigen specific, and requires the colocalization of human $\mathrm{T}$ cells, autologous human antigen-presenting cells, and antigen at the DTH challenge site in the mouse (9).

In this report we use the trans vivo DTH assay to show that a renal transplant patient who withdrew himself from immunosuppression, but retained normal graft function, has developed an active immune mechanism that inhibits donor-reactive cell-mediated immune responses. We confirm this finding in one other renal and one liver transplant recipient off all immunosuppression and show that PBMCs from these patients, as well as several others, exhibit the phenomenon of donor antigen-linked DTH nonresponsiveness, mediated by TGF- $\beta$ or IL- 10 .

\section{Methods}

PBMC isolation. Blood was sterilely collected into citrate tubes. PBMCs were isolated over Ficoll-density centrifugation according to standard methods. For trans vivo DTH analysis, PBMCs were washed three times in PBS.

Human subjects. Peripheral blood was obtained by informed consent. Patients were either transplanted at University of Wisconsin (Madison, Wisconsin, USA) or at The Ohio State University Medical Center (Columbus, Ohio, USA). Patients who discontinued their immunosuppression (D.S., J.B., M.L., R.D.) did so of their own volition at $1.5,2,2$, and 5 years after transplant, respectively. Table 1 provides clinical information on the transplant patients discussed in the manuscript, including transplant center, the type of transplant, immunosuppression, the degree of HLA mismatch from their donors, organ function, and time after transplant.

Mice. SCID mice were obtained either through Clarence Reeder at the National Cancer Institute (Bethesda, Maryland, USA) or from The Jackson Laboratory (Bar Harbor, Maine, USA) or were bred at the University of Wisconsin. All animals were housed and treated in accordance with guidelines outlined by The Ohio State University, the University of Wisconsin, and the National Institutes of Health.

Trans vivo DTH analysis. Trans vivo DTH was used to assess human cellular immunity under physiologic conditions, as described previously (9). To assess DTH reac- tivity, 6-9 million PBMCs were injected, along with antigen, into the footpads of naive SCID mice in a total volume of 20-50 $\mu \mathrm{L}$. Antigen-driven swelling was measured after 24 hours using a dial thickness gauge. Postinjection measurements were compared with preinjection measurements to obtain specific swelling. DTH reactivity is shown as the change in thickness, using units of $10^{-4}$ inches. Typical background swelling for the injection of PBMCs or antigen alone is $10-20 \times 10^{-4}$ inches. However, background levels can vary widely among responders due to high platelet contamination or previous sensitization to murine xenoantigens. Thus it is critical to monitor background swelling elicited by isolated PBMCs from each individual when injected into the footpad. When comparing responses from different patients, or when combining data from several experiments, we typically subtract the swelling elicited by injection of PBMCs alone from the swelling elicited by PBMCs plus antigen. Thus, the responses are normalized to background. When swelling responses are normalized to background, swelling less than $10 \times 10^{-4}$ inches is considered negative, responses in the range of $10-20 \times 10^{-4}$ inches are considered weak DTH responses, $25-35 \times 10^{-4}$ inches are intermediate responses, and $40 \times 10^{-4}$ inches or more are considered strong responses. Injection of antigens alone or PBS elicits swelling of less than $10 \times 10^{-4}$ inches.

Donor antigen. Donor antigen was prepared from either PBMCs or frozen splenocytes as described previously (9). Briefly, PBMCs or splenocytes were washed in sterile PBS and adjusted to a concentration of 120 million cells $/ \mathrm{mL}$. The cell mixture was sonicated using a VR50 sonicator fitted with a $2-\mathrm{mm}$ probe. The disrupted cells were centrifuged for 20 minutes at 14,000 $g$ to remove debris. The protein content of the supernatant was determined using standard methods. Ten micrograms of protein was used for each injection.

Epstein-Barrvirus antigen lysate. Epstein-Barr virus (EBV) antigen lysate was prepared from induced P3HR1 cells and was the kind gift of Bob Naegele (Viral Antigens Inc., Memphis, Tennessee, USA). This is the same EBV preparation that is used in some commercially available EBV ELISA kits and contains antigens of both lytic and latent infections. In background experiments we have determined that EBV IgG-seropositive individuals respond to the EBV lysate in the trans vivo DTH assay, whereas EBV IgG-seronegative individuals do not.

EBV-transformed lymphoblastoid cell lines. EBV-transformed lymphoblastoid cell lines (EBV-LCL) were generated by culturing PBMCs with EBV-containing B95.8 supernatant and cyclosporine (Sandoz Transplant, Basel, Switzerland), according to standard protocols (10). In some instances, purified splenic B cells were used in place of PBMCs for EBV-LCL generation, in which case, cyclosporine was not used in the tissue culture medium. All cell lines were maintained in complete DMEM containing 10\% heat-inactivated FCS (Upstate Biotechnology Inc., Saranac Lake, New York, USA). Complete medium (CM) consists of DMEM medium 
(GIBCO BRL, Grand Island, New York, USA) supplemented with $2.5 \mathrm{mM}$ HEPES buffer, $1 \mathrm{mM}$ sodium pyruvate, $2 \mathrm{mM}$ L-glutamine, $0.6 \mathrm{mM}$ arginine, $0.27 \mathrm{mM}$ L-asparagine, $0.014 \mathrm{mM}$ folic acid, $100 \mathrm{U} / \mathrm{mL}$ penicillin, $100 \mathrm{U} / \mathrm{mL}$ streptomycin (all obtained from GIBCO BRL), $0.05 \mu \mathrm{M} \beta$-mercaptoethanol (Sigma Chemical Co., St. Louis, Missouri, USA). The EBV-LCL 721.221, B62-.221, and A2-.221 were the kind gifts of Robert DeMars (University of Wisconsin) and William Hildebrand (University of Oklahoma Health Sciences Center, Oklahoma City, Oklahoma, USA). For A2-.221, the complete medium above was supplemented with $500 \mu \mathrm{g}$ G418 (Sigma Chemical Co.) to maintain transgene expression. When EBV-LCL were used as a source of antigen in the trans vivo DTH assay, cells were gammairradiated with $50 \mathrm{~Gy}$, and 0.1 million cells were coinjected with PBMCs into the footpads of SCID mice.

Antibodies. Polyclonal rabbit anti-human TGF- $\beta$ (pan specific) and control rabbit Ig's, polyclonal goat antihuman IL-10 and control goat Ig's, and monoclonal mouse IgG1 and monoclonal mouse anti-human IL-10 were all purchased from R\&D Systems (Minneapolis, Minnesota, USA). For neutralization experiments, 25 $\mu \mathrm{g}$ of the Ig's were used per injection. These concentrations were determined previously to be optimal in the mouse studies (6).

Soluble HLA antigens. Soluble HLA-A2 was purified from A2-expressing human splenocytes as described previously (11). Soluble recombinant HLA-B*1501 (B62) was purified from the supernatant of a 721.221 transfected with a truncated HLA-B* 1501 gene lacking exons 5-7 encoding the transmembrane and cytoplasmic domain (provided by William Hildebrand). Both antigens were purified by affinity chromatography over mAb-coupled Sepharose beads and were more than $90 \%$ pure as determined by SDS-PAGE analysis.

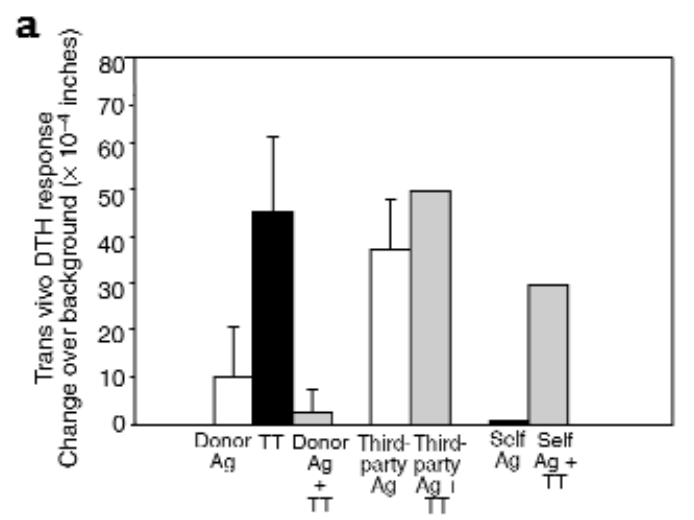

\section{Results}

Description of patients. The DTH responses of a total of 19 patients were tested in the following studies. The clinical information provided in Table 1 includes identification of the transplant center, the type of transplant, induction therapy, immunosuppression, the degree of HLA mismatch from their donors, organ function at the time of testing, and the time after transplant. As is shown in Table 1, 14 patients received kidney transplants from either living (LRD, $n=3$ ) or cadaveric (cad, $n=11$ ) donors, and 5 patients received a liver transplant. The post-transplant time ranged from 1 to 32 years, and there was a wide range of HLA disparities (0-6 mismatches). The majority of the patients presented with good graft function (OK, $n=14)$, whereas 5 patients were tested at the time of a biopsy-proven acute or chronic rejection $(n=3)$ or after the loss of graft function $(n=2)$. Of the 14 patients with good graft function, three had withdrawn themselves from all immunosuppression (D.S., R.D., M.L.), two patients were maintained on monotherapy of azathioprine (Aza; P.W.) or tacrolimus (A.J.), whereas four were maintained on dual therapy of prednisone (Pred) and calcineurin inhibitor (either cyclosporine [CsA] or tacrolimus; L.M., J.Mc., P.B., D.F.). The remaining patients with good graft function and the three patients with acute or chronic rejection were maintained on triple therapy consisting of prednisone, mycophenolate mofetil (MMF), and calcineurin inhibitor.

Description of patient D.S. Our initial studies focused on a patient who withdrew himself from immunosuppression, yet has maintained excellent graft function for 5 years since withdrawal. A Caucasian male patient, D.S., developed end-stage renal disease secondary to reflux nephropathy at the age of 17 years and underwent cadaveric renal transplantation in December 1993. The recipient and donor were serologically matched for HLA-A

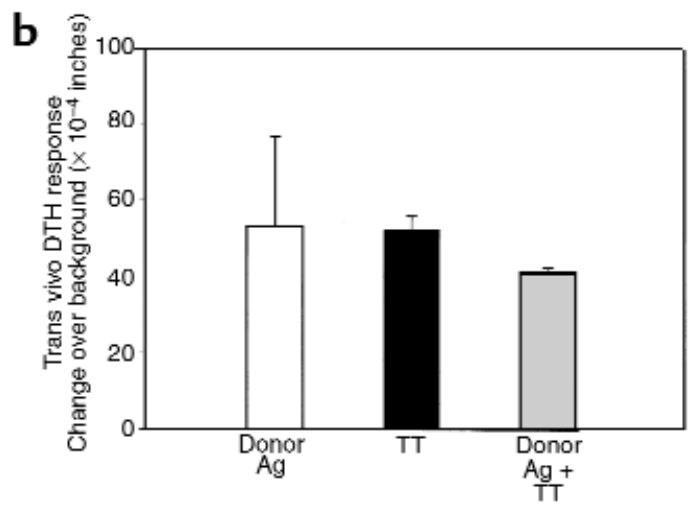

\section{Figure 1}

Trans vivo DTH responses: donor antigen-linked DTH nonresponsiveness to TT. (a) Eight million PBMCs from patient D.S. were injected into the footpads of SCID mice and the swelling measured after 24 hours. This background swelling was used to calculate the specific swelling when PBMCs plus the different antigens were included in the injection mixture. Injection of PBMCs plus self antigens (Ag) did not result in swelling, whereas injection of PBMCs plus self Ag plus $T$ resulted in swelling (not shown). These data are combined from six separate experiments performed over 8 months. (b) Eight million PBMCs from patient J.B. were injected into the footpads of SCID mice and the swelling measured after 24 hours. This background swelling was used to calculate the specific swelling when PBMCs plus donor alloantigens, $\Pi$, or donor alloantigens plus TT were included in the injection mixture. Data are combined from three separate experiments over 9 months. 
and HLA-DR, but not HLA-B alleles (HLA A1, 2 B44, 62 DR4, 13 donor $\rightarrow$ HLA A1, 2 B37, 60 DR4, 13 recipient). The recipient was treated with OKT3 mAb-induction therapy from post-transplant day 1 to day 4 , at which time his serum creatinine was $1.6 \mathrm{mg} / \mathrm{dL}$. Maintenance immunosuppression consisted of cyclosporine (6-8 $\mathrm{mg} / \mathrm{kg} /$ day), azathioprine $(150 \mathrm{mg} / \mathrm{kg} /$ day $)$, and prednisone $(30 \mathrm{mg} / \mathrm{kg} / \mathrm{day})$. The patient's postoperative course was complicated by ureteral obstruction on postoperative day (POD) 16, ultimately resulting in surgical reimplantation of the allograft ureter on POD 98 . He also had an episode of biopsy-confirmed acute rejection, for which he was successfully treated with pulse corticosteroids and OKT3 (POD 39). In early 1995, approximately 18 months after transplant, the patient discontinued all his immunosuppressive medications, but surprisingly, has retained excellent renal function (current serum creatinine: $1.2 \mathrm{mg} / \mathrm{dL}$ ). The patient underwent three biopsy procedures to evaluate his allograft: one at the time of the acute rejection episode (POD 39), one in early 1997 (year 3.2, 1.7 years after discontinuation of immunosuppression), and one in July 1998 (year $4.5,3$ years after discontinuing immunosuppression). The early biopsy (POD 39) displayed the classic features of acute rejection, a destructive infiltrate of mononuclear cells with tubulitis. In contrast, the second and third biopsies, taken during the graft acceptance phase, displayed a focal infiltrate that does not appear to be destructive or invasive. The infiltrate was confined to the interstitium, separated from nearby glomeruli and tubules, and consisted primarily of $\mathrm{CD}^{+} \mathrm{T}$ cells. The majority of the $\mathrm{CD}^{+}$cells were $\mathrm{CD}^{+}$, although mixed with a significant number of $\mathrm{CD}^{+}$cells (12). Thus, a biopsy taken after the allograft was accepted displayed a leukocytic infiltrate, but not a pattern of infiltration that suggested acute allograft rejection.

Donor-reactive alloimmunity in patient D.S. The trans vivo DTH assay was used to evaluate the DTH reactivity of patient D.S. to alloantigens and/or TT (Figure 1a).
PBMCs from patient D.S. were injected into the footpads of SCID mice either alone, with alloantigens, with TT, or with an equal mixture of both antigens. When PBMCs and donor-derived alloantigens were injected, minimal swelling was observed. In contrast, PBMCs plus TT elicited a strong swelling response. However, when PBMCs plus the combination of donor-derived alloantigens and TT are injected, the strong swelling response to TT was lost. As a specificity control, PBMCs from patient D.S. mixed with third party alloantigens (HLA-A1, --; HLA-B8, --; HLA-DR3, --) were injected into SCID mouse footpads, resulting in a moderate swelling response. Importantly, this response to third party alloantigens was not able to interfere with the TT-induced swelling response. Mixing PBMCs plus self antigens did not result in any swelling over background, and the responses to TT were in the normal range in the presence of self antigens. The inhibition of the strong TT response when donor antigens are present is indicative of an immunologic phenomenon known as bystander suppression $(13,14)$, or donor antigen-linked DTH nonresponsiveness $(5,15,16)$. For comparison, we assessed the donor-reactive DTH response from patient J.B. (HLA A2,3, B44,62 DR4, 5/1104 donor $\rightarrow$ HLA A2,32 B44,49 DR4, 5/1102 recipient). Like patient D.S., patient J.B. was transplanted at the age of 16 years and removed himself from immunosuppression within 2 years after transplant. He had good graft function for 7 years, and his biopsies also displayed focal infiltrates. However, between 9 and 10 years after transplant, patient J.B. developed a cellular acute rejection episode and lost graft function $(12,17)$. Patient J.B. was tested while on dialysis, waiting for retransplant. As seen in Figure $1 \mathrm{~b}, \mathrm{~J} . \mathrm{B}$. responded well to challenge with donor alloantigens and to TT. Importantly, the combination of donor alloantigens and TT did not result in significant suppression of the TT-reactive response. Thus, patient J.B., who rejected his allograft, had strong donor-reactive DTH responses and no donor antigen-linked DTH nonresponsiveness after graft rejection. a

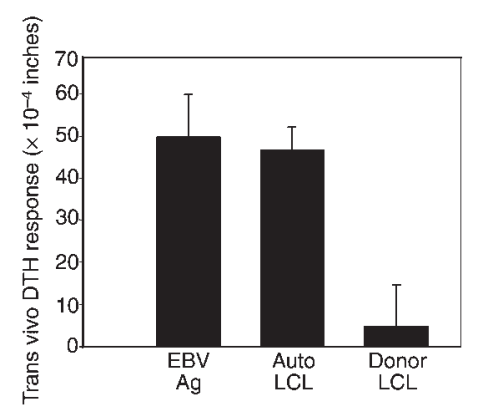

b

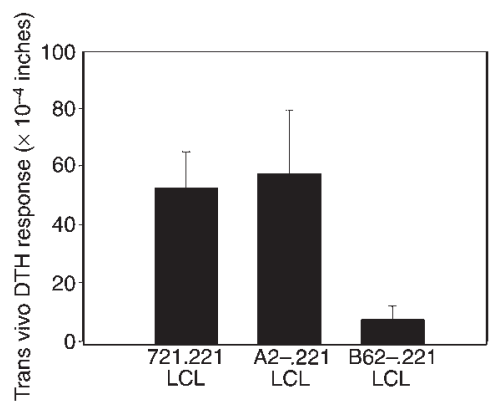

C

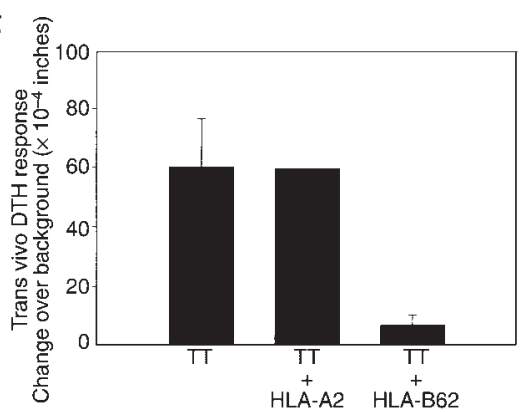

Figure 2

Specificity analysis of donor antigen-linked DTH nonresponsiveness. (a) Eight million PBMCs from patient D.S. were injected into the footpads of Balb/c SCID mice either alone or mixed with $10 \mu \mathrm{g}$ EBV lysate or $10^{5}$ autologous or donor-derived EBV-transformed LCL. Swelling was measured after 24 hours and is expressed as the change over the swelling induced by injection of PBMCs alone. Injection of EBV lysate or EBV-transformed LCL alone did not result in any increase in footpad thickness. The PBMCs also did not respond to control lysate prepared from Raji cells (not shown). (b) Eight million PBMCs from patient D.S. were injected into the footpads either alone or mixed with $10^{5}$ irradiated $721.221, \mathrm{~A} 2-$ .221, or B62-.221 LCL. Swelling was measured as described above. (c) Eight million PBMCs from patient D.S. were mixed with TT or TT plus purified HLA-A2 or HLA-B62 and injected into the footpads of SCID mice. Swelling was measured after 24 hours as described above. 
Table 1

Description of patients

\begin{tabular}{|c|c|c|c|c|c|c|c|}
\hline Patient ID & $\begin{array}{c}\text { Transplant } \\
\text { center }\end{array}$ & $\begin{array}{l}\text { Type of } \\
\text { transplant }\end{array}$ & $\begin{array}{l}\text { Graft function at } \\
\text { time of testing }\end{array}$ & $\begin{array}{c}\text { HLA mismatch } \\
\text { with donor }(A, B, D R)\end{array}$ & Induction & $\begin{array}{l}\text { Immunosuppression } \\
\text { at time of testing }\end{array}$ & $\begin{array}{l}\text { Years after } \\
\text { transplant }\end{array}$ \\
\hline D.S. & WI & Renal-cad & OK & $0,2,0$ & ОКТ3 & None, $>5 y$ & 6.5 \\
\hline R.D. & WI & Renal-LRD & OK & 0 & None & None, $>27 y$ & 32 \\
\hline P.W. & WI & Renal-LRD & OK & 0 & None & Aza only, $>6 \mathrm{mo}$ & 1 \\
\hline L.M. & $\mathrm{OH}$ & Renal-cad & OK & $1,2,2$ & OKT3 & Pred/CsA, > $2 y$ & 2.5 \\
\hline O.P. & $\mathrm{OH}$ & Renal-cad & OK & $1,2,2$ & OKT3 & Pred/CsA/MMF & 3.5 \\
\hline M.D. & $\mathrm{OH}$ & Renal-cad & OK & $2,2,2$ & Anti-IL-2R & Pred/CsA/MMF & 1 \\
\hline B.M. & $\mathrm{OH}$ & Renal-cad & OK & $2,2,2$ & OKT3 & Pred/CsA/MMF & 3.5 \\
\hline B.H. & $\mathrm{OH}$ & Renal-cad & OK & $2,2,2$ & OKT3 & Pred/CsA/MMF & 1 \\
\hline R.K. & $\mathrm{OH}$ & Renal-cad & OK & $2,2,2$ & None & Pred/CsA/MMF & 1.5 \\
\hline J.Mc. & $\mathrm{OH}$ & Renal-cad & OK & $2,2,2$ & None & Pred/CsA & 5 \\
\hline M.F. & $\mathrm{OH}$ & Renal-cad & Acute rejection & $2,1,1$ & ATG & Pred/CsA/MMF & 2.5 \\
\hline T.B. & $\mathrm{OH}$ & Renal-cad & Acute rejection & $1,2,2$ & Anti-IL-2R & Pred/CsA/MMFA & 1 \\
\hline G.R. & $\mathrm{OH}$ & Renal-cad & Graft loss & $1,2,2$ & OKT3 & None & 1 \\
\hline J.B. & WI & Renal-LRD & Graft loss & $1,1,0$ & DST & None & 10 \\
\hline M.L. & WI & Liver & $\mathrm{OK}^{\mathrm{B}}$ & $0,2,2$ & None & None, $>3 y$ & 5 \\
\hline A.J. & WI & Liver & OK & $2,2,2$ & None & Tacrolimus, > $6 \mathrm{mo}$ & 3.5 \\
\hline P.B. & WI & Liver & OK & $1,2,0$ & None & Pred/tacrolimus & 4 \\
\hline D.F. & $\mathrm{OH}$ & Liver & OK & $1,2,2$ & ALG & Pred/CsA & 13 \\
\hline J.W. & WI & Liver & Chronic rejection & $1,1,2$ & None & Pred/tacrolimus/MMF & 3 \\
\hline
\end{tabular}

APatient's immunosuppression changed (substitute tacrolimus for CsA) after acute rejection episode and renal dysfunction. ${ }^{B}$ Some bile duct-related dysfunction (elevated GG-T) secondary to fatty deposits in liver. All biopsies are consistently rejection negative.

HLA specificity analysis of donor antigen-linked nonresponsiveness in patient D.S. Patient D.S. is IgG seropositive for $\mathrm{EBV}$, indicating EBV-reactive $\mathrm{T}$ cells are present and functional. Thus, his PBMCs should respond in the trans vivo DTH assay to EBV antigens expressed by autologous or donor-derived EBV-LCL. Since LCL express several EBV proteins (although not viral capsid antigens) that are antigenic in their own right, we first tested for the patient's recall response to EBV antigens with a commercially available lysate prepared from induced P3HRY cells (gift of Bob Naegele, Viral Antigens Inc.), which contains antigens from both lytic and latent infection. As shown in Figure 2a, PBMCs from D.S. responded strongly to $10 \mu \mathrm{g}$ EBV lysate. This response was equal in magnitude to the response induced by $10^{5}$ irradiated autologous LCL, indicating that patient D.S. is sensitized to EBV antigens expressed by LCL. In contrast, patient D.S. did not respond to donor-derived EBV-transformed LCL. Additional experiments showed that the donor-derived LCL could elicit strong DTH responses by PBMCs from other EBV-reactive individuals (not shown). Thus, patient D.S. did not respond to EBV antigens when they were colocalized with donor alloantigens, providing another example of donor antigen-linked DTH nonresponsiveness.

To further evaluate the donor HLA antigens that trigger donor antigen-linked DTH non-responsiveness, we used the mutant LCL 721.221, which has lost classic HLA class I (HLA-A, -B, -C) expression, as well as variants that have been transfected with genes encoding specific HLA molecules. The mutant line, 721.221, expresses HLA DR1, but not HLA-A, $-\mathrm{B}$, or $-\mathrm{C}$, antigens. In addition to HLA-DR1, the transfected lines express either HLA-A2
(A*201) (cell line A2-.221) or HLA-B62 (B*1501) (cell line B62-.221). Both D.S. and his allograft donor share HLAA2, whereas only the allograft donor displays HLA-B62. As shown in Figure 2b, PBMCs from D.S. responded well to EBV antigens expressed by the parent line, 721.221, and by the HLA-A2-transfected line, A2-.221. However, they did not respond to the HLA-B62-transfected cell line. The absence of DTH responses was not due to a lack of EBV antigen expression, since other EBV-reactive individuals responded well to this line (not shown). Thus, these data demonstrate that the donor antigen-linked DTH nonresponsiveness expressed by patient D.S. can be triggered by a single HLA class I alloantigen (HLA-B62) and that this alloantigen is sufficient to drive the DTH nonresponsiveness to unrelated EBV antigens.

Because the LCL may act as antigen-presenting cells in trans vivo DTH responses, it is possible that the donor antigen-linked nonresponsiveness could be triggered through either the direct and/or the indirect alloantigen presentation pathways (reviewed in ref. 18). Therefore, we tested whether purified, cell-free HLAB62, when mixed with the recall antigen, TT, was sufficient to block the TT-reactive DTH response. In this experiment, the only antigen-presenting cells present are of recipient origin, forcing indirect presentation of the antigens. Similar to the experiments in Figure 1a, PBMCs from patient D.S. were mixed with TT alone or a combination of TT and either purified HLA-A2 or HLA-B62, then injected into the footpads of naive SCID mice. Swelling was assessed after 24 hours. As observed previously, PBMCs from D.S. respond strongly to TT. However, the addition of HLA-B62 completely suppressed the TT-reactive DTH response. The addi- 
Table 2

Donor antigen-linked nonresponsiveness in transplant patients ${ }^{\mathrm{A}}$

\begin{tabular}{|c|c|c|c|c|c|c|}
\hline \multicolumn{7}{|c|}{ Donor antigen-linked DTH nonresponsiveness to third-party antigens } \\
\hline REGULATED PHENOTYPE & Patient & Graft function & Immunosuppression & Donor Ag & Recall AgB & Donor $\mathrm{Ag}+$ recall $\mathrm{Ag}$ \\
\hline \multirow[t]{5}{*}{ Renal transplant } & D.S. & OK & None & 10 & 30 & 0 \\
\hline & R.D. & OK & None & 0 & 45 & 10 \\
\hline & P.W. & OK & Aza & 15 & 40 & 5 \\
\hline & L.M. & OK & Pred/CsA & 10 & 30 & 10 \\
\hline & O.P. & OK & Pred/CsA/MMF & 0 & 40 & 0 \\
\hline \multirow[t]{2}{*}{ Liver transplant } & M.L. & OK & None & 15 & 35 & 5 \\
\hline & A.J. & OK & Tacrolimus & 5 & 40 & 5 \\
\hline \multicolumn{7}{|l|}{ NONREGULATED PHENOTYPE } \\
\hline \multirow[t]{9}{*}{ Renal transplant } & M.D. & OK & Pred/CsA/MMF & 10 & 60 & 80 \\
\hline & J.Mc. & OK & Pred/CsA & 0 & 50 & 70 \\
\hline & R.K. & OK & Pred/CsA/MMF & 20 & 30 & 40 \\
\hline & B.H. & OK & Pred/CsA/MMF & 100 & 60 & 100 \\
\hline & B.M. & OK & Pred/CsA/MMF & 90 & 65 & NT \\
\hline & M.F. & $\mathrm{AR}^{\mathrm{C}}$ & Pred/CsA/MMF & 60 & 100 & NT \\
\hline & T.B. & $\mathrm{AR}^{\mathrm{C}}$ & Pred/CsA/MMF & 25 & 20 & NT \\
\hline & J.B. & Graft loss & None & 40 & 50 & 40 \\
\hline & G.R. & Graft loss & None & 60 & 50 & NT \\
\hline \multirow[t]{3}{*}{ Liver transplant } & P.B. & OK & Pred/tacrolimus & 45 & 45 & 45 \\
\hline & D.F. & OK & Pred/CsA & 5 & 30 & 40 \\
\hline & J.W. & $\mathrm{CR}^{\mathrm{D}}$ & Pred/tacrolimus/MMF & 40 & 40 & 30 \\
\hline
\end{tabular}

${ }^{A}$ Data shown are from one representative experiment, with PBMC-alone background subtracted. Interpretation of responses (as in Methods): < 10, negative; 10-20, weak; 20-40, moderate; > 40, strong. ${ }^{B}$ Recall antigen is either TT or EBV. CAcute rejection. ${ }^{D}$ Chronic rejection. NT, not tested.

tion of HLA-A2 to PBMCs plus TT had no effect on the TT-reactive DTH response. Thus, in the case of HLA Class I mismatched allograft acceptance, the addition of a purified donor HLA molecule (HLA-B62) is sufficient to trigger immune regulation (Figure 2c).

Cytokine neutralization uncovers donor-reactive DTH responses. In murine models of allograft acceptance, donor-reactive DTH responses can be restored if TGF- $\beta$ or IL-10 is neutralized at the DTH site (6). Both TGF- $\beta$ and IL-10 have been reported to have immunosuppressive properties and be involved in allograft acceptance $(6$, 19-30). We therefore wished to determine if serologic neutralization of either of these cytokines could uncover donor-reactive responses in the human allograft acceptor, D.S. PBMCs from patient D.S. were mixed with donor alloantigens and Ig's specific for either TGF- $\beta$ or IL-10 and injected into the footpads of SCID mice (Figure 3). Control injections used nonimmune Ig. No swelling over background was detected when control Ig was included in the injection mixture, again demonstrating the lack of DTH responses by D.S. to donor alloantigens. However, when anti-TGF- $\beta$ antibodies were present, significant swelling was observed in response to donor alloantigens. A similar unmasking of responses was observed when irradiated donor-derived EBV-transformed LCL were used as the antigen source. In contrast, no swelling was elicited with either anti-IL-10 antibodies or when antibodies specific for TGF- $\beta$ were mixed with autologous self antigens. These data indicate that patient D.S. is sensitized for DTH responses to donor alloantigens, but that these responses are impaired by a regulatory mechanism that involves local TGF- $\beta$ activity.
A different pattern of immune regulation was observed when PBMCs from patient A.J. were tested in the trans vivo DTH assay. Patient A.J. is a diabetic 57year-old male who received a six-HLA antigen-mismatched liver transplant in 1995 (HLA A30,74 B7,42 DR7,8 donor $\rightarrow$ HLA A2,24 B44,50 DR4 recipient). Patient A.J. underwent steroid withdrawal in 1999 and is currently maintained on FK506 with excellent graft function (aspartate aminotransferase, $30 \mathrm{U} / \mathrm{L}$; alanine aminotransferase, $46 \mathrm{U} / \mathrm{L}$ ). The responses of PBMCs from A.J. in cytokine neutralization experiments are shown in Figure 3b. PBMCs plus donor antigens and either control or cytokine-specific antibodies were injected into the footpads of naive SCID mice. Swelling was measured after 24 hours. No significant swelling was detected when donor antigens and control Ig were included in the injection mixture. Similar results were obtained with donor antigen alone, demonstrating a lack of donor-reactive DTH. In contrast to the results observed previously with patient D.S., PBMCs from patient A.J. did not exhibit donorreactive DTH responses when neutralizing antibodies to TGF- $\beta$ were included in the injection mixture. However, strong donor-reactive DTH responses were detected when neutralizing antibodies to IL-10 were included. As a control, PBMCs from A.J. were mixed with self antigens and either control or anti-IL-10 antibodies. No significant swelling was induced with self antigens, nor did anti-IL-10 uncover any response to self antigens. Thus, it appears that donor-reactive DTH responses can be uncovered by neutralizing local activity of either TGF- $\beta$ (D.S.) or IL-10 (A.J.). 
Trans vivo DTH analysis in renal and liver transplant recipients: donor antigen-linked nonresponsiveness is a common feature of allograft acceptance. We next wished to determine if the responses observed with patient D.S. showing donor antigen-linked nonresponsiveness were unique to this individual or if similar DTH regulation could be observed in other transplant patients, particularly patients with good graft function who are off all immunosuppression. We also wished to determine if the pattern of nonregulated responses after graft rejection, observed with patient J.B., were also observed in other patients who have experienced acute or chronic rejection. We used the trans vivo DTH assay to assess donor-reactive DTH responses and DTH responses to recall antigens (TT or EBV) in all 19 patients (Table 2), and we were able to assess donor antigen-linked nonresponsiveness in 15 of these patients. Ten of the 19 patients demonstrated low to absent donor-reactive DTH responses, along with moderate to strong DTH responses to recall antigens. When both donor antigens and recall antigens were combined, the response to recall antigens was suppressed in seven of the 10 low responders. We classify these patients who exhibit donor antigen-linked nonresponsiveness as having the regulated phenotype. Importantly, all three patients who had completely removed themselves from immunosuppression (D.S., R.D., M.L.) were in this group. Interestingly, three of the remaining four patients were maintained on reduced immunosuppression (P.W., A.J., L.M.). One patient of the seven receives triple therapy of prednisone, cyclosporine, and mycophenolate mofetil (O.P.). Thus, the regulated phenotype observed in D.S. may be a common feature of clinically tolerant transplant patients.

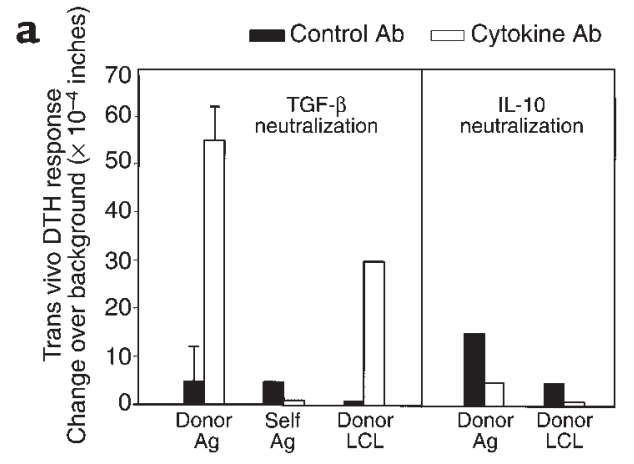

Patients whose PBMCs exhibited moderate to strong DTH responses to donor antigens as well as to recall antigens, or weak donor-reactive responses but no donor antigen-linked nonresponsiveness, are classified as having the nonregulated phenotype. These 12 patients included two who had lost graft function (J.B., G.R.) and three patients who were tested during acute or chronic rejection (M.F., T.B., J.W.). These five patients all exhibited moderate to strong donor-reactive DTH responses. The other seven patients exhibiting the nonregulated phenotype have maintained good graft function. Four of these have exhibited donor-reactive DTH responses (R.K., B.H., B.M., P.B.), indicating that sensitization to donor antigens does not preclude good graft function in patients maintained on immunosuppression. Finally, three patients had weak or absent donorreactive DTH responses, but did not exhibit donor antigen-linked nonresponsiveness (M.D., J.Mc., D.F.).

Effect of cytokine neutralization on donor-reactive DTH responses in transplant patients. Six of the 7 patients in Table 2, whose PBMCs exhibited donor antigen-linked nonresponsiveness (the regulated phenotype), were tested to determine if cytokine neutralization would uncover donor-reactive DTH responses. The effects of cytokine neutralization on donor-reactive DTH responses for these seven transplant patients are shown in Table 3. Like patient D.S., serologic neutralization of TGF- $\beta$ uncovered donor-reactive DTH responses in PBMCs from renal recipients R.D. and P.W. and liver recipient M.L. However, blockade of TGF- $\beta$ at the DTH challenge site did not uncover donor-reactive responses in patient A.J. As noted previously (Figure $3 \mathrm{~b}$ ), serologic neutralization of IL-10, but not TGF- $\beta$, uncovered donor-reactive DTH responses in PBMCs from patient A.J. Patient

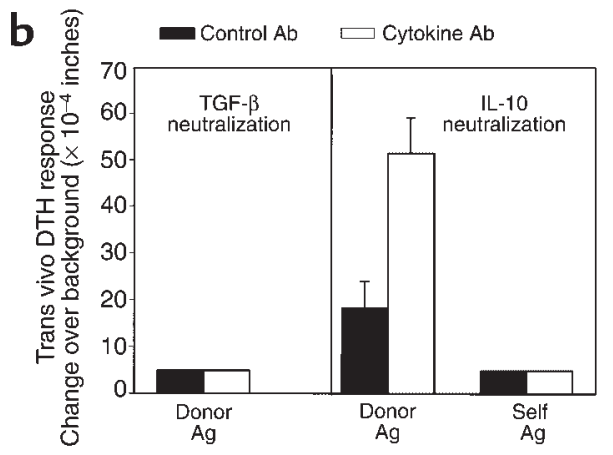

Figure 3

Effect of cytokine neutralization on donor-reactive DTH. (a) Eight million PBMCs from patient D.S. were mixed with $10 \mu \mathrm{g}$ donor or self antigen or $10^{5}$ irradiated donor-derived EBV-transformed LCL and $25 \mu \mathrm{g}$ of either control rabbit IgG or rabbit anti-human TGF- $\beta$ IgG and injected into the footpads of Balb/c SCID mice. Swelling was measured after 24 hours. Results are expressed as the change over the swelling induced by injection of PBMCs alone. To determine the effect of neutralizing antibodies to IL-10, 8 million PBMCs from patient D.S. were mixed with $10 \mu \mathrm{g}$ donor antigen or $10^{5}$ irradiated donor-derived EBV-transformed LCL and $25 \mu \mathrm{g}$ of either control goat IgG or goat antihuman IL-10. Swelling was measured after 24 hours, as described above. (b) Eight million PBMCs from patient A.J. were mixed with $10 \mu \mathrm{g}$ donor antigens and $25 \mu \mathrm{g}$ of either control rabbit IgG or rabbit anti-human TGF- $\beta$ IgG and injected into the footpads of SCID mice. Swelling was measured as described above. To assess the effect of neutralizing antibodies to IL-10, PBMCs from patient A.J. were mixed with donor or self antigens and $25 \mu \mathrm{g}$ of either control Ig or anti-IL-10. The results shown are from three separate experiments. In two experiments, the anti-IL-10 was a mAb with a mouse IgG-1 control. In the third experiment, goat anti-human IL-10 and goat IgG were used as the specific antibody and control, respectively. The results were not significantly different whether the polyclonal antibody or mAb was used. 
Table 3

Effect of cytokine neutralization on donor-reactive DTH in transplant patients ${ }^{\mathrm{A}}$

\begin{tabular}{lcccc} 
& \multicolumn{5}{c}{ Cytokine neutralization } \\
Patient & $\begin{array}{c}\text { Donor Ag + } \\
\text { CTRL Ab }\end{array}$ & $\begin{array}{c}\text { Donor Ag + } \\
\text { anti-TGF- } \beta\end{array}$ & $\begin{array}{c}\text { Donor Ag + } \\
\text { CTRL Ab }\end{array}$ & $\begin{array}{c}\text { Donor Ag + } \\
\text { anti-IL-10 }\end{array}$ \\
D.S. & 0 & 40 & 5 & 0 \\
R.D. & 0 & 30 & NT & NT \\
M.L. & 5 & 35 & NT & NT \\
P.W. & 0 & 30 & 0 & 5 \\
A.J. & 5 & 5 & 15 & 60 \\
L.M. & 10 & 20 & 0 & 10 \\
\hline
\end{tabular}

${ }^{A}$ Data shown are from one representative experiment, with PBMC-alone background subtracted. Interpretation of responses (as in Methods): $<10$, negative; $10-20$, weak; $20-40$, moderate; $>40$, strong. NT, not tested.

L.M. recovered weak donor-reactive DTH responses when either TGF- $\beta$ or IL-10 was neutralized. These data suggest that various types of immune regulation can limit donor-reactive DTH responses in patients with allograft acceptance. These pathways may use TGF- $\beta$, IL10 , or both cytokines simultaneously.

\section{Discussion}

Clinical transplantation has become an accepted therapy for many medical conditions, although there is little understanding of the immune events that occur in transplant patients as they evolve a relationship with their graft alloantigens. It is clear that some patients develop an aggressive relationship with their graft alloantigens, as evidenced by the occurrence of acute rejection episodes (cellular sensitization) or the development of donor-reactive alloantibodies (humoral sensitization). However, about half of all transplant patients do neither. Further, the recipient/graft relationship is not necessarily static, but evolves over time. Whereas many patients who withdraw from immunosuppression develop acute rejection, some do not. These latter patients may provide clues to human allograft acceptance.

Although rarely observed in humans, long-term, drug-free allograft acceptance is commonly induced in rodent transplant models. In contrast to acute rejection, which is commonly associated with the development of both donor-reactive alloantibodies (humoral sensitization) and donor reactive DTH responses (cellular sensitization) $(5,31-34)$, allograft acceptance has been associated with variable alloantibody production and a lack of donor-reactive DTH responses $(5,31)$. In rodent models of transplantation, allograft acceptance is also often associated with the phenomenon known as donor antigen-linked nonresponsiveness (LNR) (5, $6,16,35)$ and the related phenomenon of infectious tolerance $(15,36-38)$. Both of these phenomena appear to be active processes that are dependent upon donor antigen and T cells $(37,38)$. Data demonstrating LNR have been obtained by both transplanting specific organs (heart, skin, kidney, cornea) and by using DTH as a surrogate test for donor-specific cellular immunity $(5,37-40)$. We have demonstrated that splenocytes from cardiac allograft acceptor mice can actively downregulate a TT-reactive DTH response, provided that both donor antigen and TT are present at the same site, thus demonstrating donor antigen-linked nonresponsiveness. The spontaneous acceptance of many corneal allografts is also associated with a lack of systemic DTH responses to the intraocularly administered antigens. This phenomenon is termed anterior chamber-associated immune deviation (ACAID) (39) and also exhibits the property of LNR (16). Hutchinson and Morris (41) first demonstrated donor-antigen LNR to organ transplants by showing that DA strain rats tolerized to LEW alloantigens can accept an F1 transplant bearing both donor (LEW) and third-party alloantigens. Hutchinson et al (35) demonstrated that tolerance to haptens could protect haptenated renal allografts, again demonstrating linked nonresponsiveness.

This report describes a similar protective immune mechanism in human transplant patients. The traditional method of DTH skin testing has been avoided in the past because deposition of subcutaneous alloantigen has the potential to drive T-cell sensitization. We recently developed the trans vivo DTH method, a method of DTH analysis in which human PBMCs plus challenge antigen are injected into the footpad or ear of a naive mouse (9). We have determined that swelling occurs only when the PBMC donor has been previously sensitized to the antigens. In this report we use the trans vivo DTH assay to perform an in-depth assessment of the donorreactive DTH responses of a clinically tolerant transplant patient, D.S. Patient D.S. discontinued immunosuppressive therapy 5 years ago, yet continues with excellent renal function and no acute rejection episodes. We demonstrate that this patient not only fails to exhibit donor-reactive DTH responses, but also exhibits donor antigen-linked DTH nonresponsiveness. Patient D.S. does respond by DTH to two nominal antigens, TT and EBV, demonstrating that his $\mathrm{T}$ cells are not generally impaired. However, when either TT or EBV antigens are colocalized with donor alloantigens, the DTH responses to these antigens are diminished (Figures 1, 2, and 3). This finding was confirmed in studies of two other graft recipients (R.D., M.L.) with good graft function without immunosuppression. Patient R.D., now 33 years after transplant, is one of the earliest documented cases of immunosuppression cessation (4). Patient M.L., currently 6 years after transplant, is an example of the phenomenon of clinical liver transplant tolerance (1) (Table 2). Importantly, we have demonstrated that a single donor class I HLA antigen, HLA-B62, in either membrane-bound or soluble form, can drive this linked nonresponsiveness in PBMCs from D.S. (Figure 2, b and c). No donor antigen-linked nonresponsiveness was observed in patient J.B., who had also discontinued all immunosuppression but at the time of testing had rejected his transplant (Figure $1 \mathrm{~b}$ ), nor in patients undergoing acute or chronic rejection (Table 2). 
Two patients in the study, R.D. and P.W., are HLA identical with their donors, yet exhibit donor antigen-linked nonresponsiveness to EBV and TT. This suggests that donor minor histocompatibility antigens can trigger immune regulation. Indeed, a minor histocompatibility antigen difference at the HA-1 locus has been identified between patient R.D. and her donor (J.M. Lee, E. Jankowska-Gan, and W.J. Burlingham, manuscript in preparation). Indeed, the original studies demonstrating donor antigen-linked nonresponsiveness by Davies et al. (15) were performed in an MHC-identical murine allograft system in which allograft rejection is triggered by multiple minor histocompatibility antigen differences. Recently, Valujskikh et al. (42) have shown that a transgenic minor antigen can trigger rejection and that tolerance to this minor antigen is associated with graft acceptance through a dominant-negative regulatory pathway (A. Valujskikh et al., manuscript submitted for publication).

The immunoregulatory cytokine TGF- $\beta$ can block DTH responses and prolong graft survival in experimental animals $(6,25-27,43,44)$. In addition, IL-10 has also been implicated in controlling cellular immune responses, including DTH and allograft rejection $(6,27-29,45)$. Recent studies suggest that the generalized suppression in donor antigen-linked DTH nonresponsiveness is caused by the local activity of TGF- $\beta$ or IL-10 by donorreactive T cells $(6,25-27,44)$ (A. Valujskikh et al., manuscript submitted for publication). We demonstrate here that the donor antigen-linked DTH nonresponsiveness in PBMCs from patient D.S. can be subverted by neutralizing local TGF- $\beta$ with specific antibodies. Under these conditions, donor-reactive DTH responses are expressed (Figure 3). This observation indicates that the patient is, indeed, sensitized for DTH responses to donor alloantigens, but that this DTH response is superceded by a local, antigen-induced regulatory mechanism that involves TGF- $\beta$. Similar results were observed with PBMCs from patients R.D., M.L., and P.W. (Table 3), all of whom appear to have latent donor-reactive DTH responses uncovered by anti-TGF- $\beta$. In contrast, patient A.J. exhibited donor-reactive DTH responses when IL-10, but not TGF- $\beta$, was neutralized. In addition, weak donor-reactive DTH responses in PBMCs from patient L.M. were uncovered by neutralization of either TGF- $\beta$ or IL-10. It is intriguing that two separate mechanisms may be operable, suggesting that allograft acceptance may be associated with alternative regulatory mechanisms. The factors that determine the selection of regulatory pathway are currently unknown. One factor may be the choice of immunosuppressive strategy. Since both A.J. and L.M. receive calcineurin inhibitors, these types of immunosuppression may influence the pathway of immune regulation. Our laboratories are currently investigating what factors may contribute to the preferential use of one or the other of these cytokines.

Liver allografts are historically considered to be more easily accepted than other organs in both animal models and humans (46-48). One suggested mechanism of acceptance is the ultimate deletion of donor-reactive $\mathrm{T}$ cells $(49,50)$. If patients have deleted their donor-reactive cells, no donor antigen-linked nonresponsiveness would be detectable, nor would any donor-reactive DTH be recovered by cytokine neutralization. It is intriguing to postulate that patient D.F. may be one such patient, it has been 13 years since this patient's transplant, yet the patient exhibits no donor-reactive DTH nor donor antigen-linked nonresponsiveness (Table 2). However, this is clearly not the case with patients M.L. or A.J., since immune regulation is operative in these patients. Indeed, donor-reactive T-cell functions can often be detected in liver allograft acceptance $(51,52)$, suggesting that multiple mechanisms may account for it.

These data in the human transplant patients are in agreement with our earlier findings in inbred mice in which antibodies to TGF- $\beta$ or IL-10 could uncover donor-reactive DTH responses in cardiac allograft-acceptor animals $(5,6)$ and skin graft-acceptor animals (A. Valujskikh et al., manuscript submitted for publication) as well as those of Josien and Verbanac $(43,44)$, where treatment of allograft acceptor animals with antibodies to TGF- $\beta$ resulted in graft rejection. In addition, mRNA specific for TGF- $\beta$ was observed in all of these different allograft systems. In the case of patient D.S., biopsy mRNA analysis was consistent with the cytokine regulation pattern seen in the trans vivo DTH analysis; i.e., mRNA for TGF- $\beta$ is present in biopsy tissue from patient D.S., but IL-10 mRNA was absent (53). The results with patient A.J., in which neutralization of IL-10 but not TGF- $\beta$ uncovered donor-reactive DTH responses, is similar to the situation we have observed in B-cell knockout recipients of cardiac allografts. These animals can be induced to accept an allograft, lack donor-reactive DTH responses, and exhibit donor antigen-linked nonresponsiveness. However, neutralization of IL-10, but not TGF- $\beta$, uncovers donor-reactive DTH (A.A. Bickerstaff et al., manuscript in preparation).

The ability to uncover donor-reactive DTH responses by neutralizing TGF- $\beta$ or IL-10 highlights a potentially hazardous situation. The clinically tolerant patients in this study (D.S., R.D., M.L.) were all sensitized to donor alloantigens in both destructive (inflammatory) and protective (anti-inflammatory) pathways. Currently, the protective pathway is dominant and blocks donor-reactive DTH responses and possibly acute rejection. The relationship between patients and their graft is likely a dynamic one and may change over time. Should the protective pathway be overridden, for example, by infection or by a sudden loss of T-cell clones that may be essential for the protective pathway (17), the destructive pathway is fully functional and leaves these patients susceptible to acute rejection (discussed in ref. 12). We now have preliminary data that indicates that the recovery of donor-reactive DTH responses in patient J.B. preceded cellular rejection and graft loss (W.J. Burlingham et al., manuscript in preparation). Indeed, donor-reactive DTH responses are detected in patients who lost their grafts subsequent to acute rejection (J.B., G.R.), are in 
the midst of an acute rejection (M.F., T.B.), or have chronic rejection (J.W.). Interestingly, we have also detected donor-reactive DTH responses in patients with good graft function (Table 2). These data would indicate that these patients have become sensitized to their graft antigens and may be at increased risk for acute rejection if their immunosuppression levels decrease. Finally, we observed three patients (M.D., J.Mc., D.F.) who are maintained on immunosuppression and do not respond to donor alloantigens, nor do they exhibit donor antigen-triggered nonresponsiveness to recall antigens. These patients may have deleted antidonor Tcell clones. Alternatively, these patients may have avoided sensitization because of the immunosuppression regimen and should the immunosuppression decrease or be removed, may become sensitized to alloantigens. The establishment and maintenance of donor antigen-linked nonresponsiveness could also be used as a marker for analysis of patients being considered for or already enrolled in immunosuppression withdrawal trials, particularly since the regulated phenotype can develop in patients still on immunosuppression.

Acute rejection episodes can often be reversed by treatment with immunosuppressive agents such as OKT3 or prednisone, but successful reversal of acute rejection may not mean reversal of donor-reactive immunologic sensitization. To date, this area has received little study, in part due to the lack of testing systems. The trans vivo DTH assay can now be used to investigate donor-antigen sensitization in transplant patients before, during, and after treatment for acute rejection. In patient D.S., the uncovering of strong DTH responses to donor alloantigens after neutralization of TGF- $\beta$ suggests that T-cell sensitization had occurred in the course of his early acute rejection and remained after its reversal. Similar acute rejection episodes prior to prolonged graft acceptance without immunosuppression have been noted in patients M.L. (unpublished observation) and J.B. (2). It is intriguing to hypothesize that such an acute rejection episode may be beneficial in setting up graft acceptance mechanisms in some patients.

In summary, we have performed an in-depth dissection of donor-reactive immune responses in three patients who maintain excellent graft function in the absence of immunosuppression and are thus clinically tolerant. This excellent graft function is associated with a lack of donor-reactive DTH responses, due to overriding immune regulation mediated by TGF- $\beta$. In addition, three patients maintained on immunosuppression also exhibit immune regulation mediated by TGF- $\beta$. One patient (A.J.), who continues to have excellent graft function on tacrolimus monotherapy, exhibits IL-10-mediated, rather than TGF- $\beta$-mediated, regulation. This indicates that alternative pathways may exist for the regulated phenotype. The relationship between donor antigen-linked DTH nonresponsiveness and immunosuppression-free graft acceptance requires further study.
These data suggest several possibilities. First, donor antigen-linked DTH nonresponsiveness as a graft acceptance mechanism may be secondary to other mechanisms such as apoptosis and anergy (54-60) and is simply an interesting surrogate marker for allograft acceptance. Second, clinical tolerance may be associated with and caused by active immune regulation characterized by donor antigen-linked DTH nonresponsiveness (bystander suppression) mediated in part by TGF- $\beta$ or IL-10. Third, donor antigen-linked DTH nonresponsiveness may not be necessarily rare and confined to unusual individuals who accept their grafts without immunosuppression. If so, assessment of donor-reactive DTH and donor antigen-linked DTH nonresponsiveness may prove extremely valuable for following the evolving immune relationship between recipients and their graft. Indeed, this assay may help identify patients who could benefit from immunosuppression reduction trials. Rational, systematic, long-term assessment of acute rejection, donor-specific sensitization (both humoral and cell mediated), and clinical tolerance must be performed in large numbers of transplant patients to prove or disprove these hypotheses.

\section{Acknowledgments}

The authors would like to thank Marsha Stalker for her secretarial assistance; Sheila Zierkle for administrative assistance; and Vanita Malik, Maria Bellizzi, Patrice Hennessy, Michelle Vermillion, and John Wilson for their expert technical assistance. The authors also thank Viral Antigens Inc. for providing the EBV lysate used in these studies. The authors also thank Ronald M. Ferguson, Hans Sollinger, Stuart Knechtle, W. Jim Waldman, and Alice Bickerstaff for helpful discussions. This is manuscript 137 from the Transplant Sciences Program of The Ohio State University College of Medicine and Public Health. This work was supported, in part, by the National Institutes of Health (NIH) grants P30-CA16058 (A.M. VanBuskirk and C.G. Orosz), R29AI40909 (A.M. VanBuskirk), R01-HL61966, P01AI/HL40150 (C.G. Orosz), and K02-AI01452, R01AI44077, R01-DK50954 (W.J. Burlingham), and the Medical Research Development Fund of the Department of Surgery at The Ohio State University (A.M. VanBuskirk and R.P. Pelletier).

\footnotetext{
1. Starzl, T.E., et al. 1993. Cell migration and chimerism after whole-organ transplantation: the basis of graft acceptance. Hepatology. 17:1127-1152.

2. Burlingham, W.J., et al. 1995. Microchimerism linked to cytotoxic T lymphocyte functional unresponsiveness (clonal anergy) in a tolerant renal transplant recipient. Transplantation. 59:1147-1155.

3. Zoller, K.M., Cho, S.I., Cohen, J.J., and Harrington, J.T. 1980. Cessation of immunosuppressive therapy after successful transplantation: a national survey. Kidney Int. 18:110-114.

4. Uehling, D.T., Hussey, J.L., Weinstein, A.B., Wank, R., and Bach, F.H. 1976. Cessation of immunosuppression after renal transplantation. Surgery. 79:278-282.

5. VanBuskirk, A.M., Wakely, M.E., Sirak, J.H., and Orosz, C.G. 1998. Patterns of allosensitization in allograft recipients: long-term cardiac allograft acceptance is associated with active alloantibody production in conjunction with active inhibition of alloreactive delayed-type hypersensitivity. Transplantation. 65:1115-1123.

6. Bickerstaff, A., VanBuskirk, A., Wakely, M., and Orosz, C. 2000. Transforming growth factor-beta and interleukin-10 subvert alloreactive
} 
delayed type hypersensitivity in cardiac allograft acceptor mice. Transplantation. 69:1517-1520.

7. Bouma, G.J., van der Meer-Prins, E.M.W., Van Der Woude, F.J., van Rood, J.J., and Claas, F.H.J. 1995. Relevance of pretransplant donor-specific T cell allorepertoire for human kidney graft survival. Transplantation. 59:969-976.

8. Vaessen, L.M.B., et al. 1994. Differential avidity and cyclosporine sensitivity of committed donor-specific graft-infiltrating cytotoxic T cells and their precursors. Transplantation. 57:1051-1059.

9. Carrodeguas, L., et al. 1999. Trans vivo analysis of human delayed-type hypersensitivity reactivity. Hum. Immunol. 60:640-651.

10. Tosato, G. 1996. Generation of Epstein-Barr Virus (EBV)-immortalized B cell lines. In Current protocols in immunology. Volume 2. J. Coligan, A. Kruisbeek, E. Shevach, and W. Strober, editors. John Wiley \& Sons Inc. New York, New York, USA. 7.22.1-7.22.3.

11. DeVito, L.D., Sollinger, H.W., and Burlingham, W.J. 1990. Adsorption of cytotoxic anti-HLA antibodies with HLA class I immunosorbant beads. Transplantation. 49:925-931.

12. Burlingham, W.J., et al. 1999. Focal infiltrates in kidney allografts: developing a model for clinical transplant peripheral tolerance. Graft. 2:253-260.

13. Prop, J., Hutchinson, I.V., and Morris, P.J. 1986. Induction and persistence of suppression of contact hypersensitivity against bystander haptens and alloantigens in rats. Cell. Immunol. 99:85-94.

14. Sirak, J.H., Orosz, C.G., Roopenian, D.C., Wakely, E., and VanBuskirk, A.M. 1998. Cardiac allograft tolerance. Failure to develop in interleukin4-deficient mice correlates with unusual allosensitization patterns. Transplantation. 65:1352-1356.

15. Davies, J.D., Leong, L.Y.W., Mellor, A., Cobbold, S.P., and Waldmann, H. 1996. T cell suppression in transplantation tolerance through linked recognition. J. Immunol. 156:3602-3607.

16. Niederkorn, J.Y., Mayhew, E., and He, Y. 1995. Alloantigens introduced into the anterior chamber of the eye induce systemic suppression of delayed hypersensitivity to third-party alloantigens through "linked recognition". Transplantation. 60:348-354.

17. Kusaka, S., et al. 2000. Clonotype analysis of human alloreactive T cells: a novel approach to studying peripheral tolerance in a transplant recipient. J. Immunol. 164:2240-2247.

18. Gould, D.S., and Auchincloss, H.J. 1999. Direct and indirect recognition: the role of MHC antigens in graft rejection. Immunol. Today. 20:77-82.

19. Groux, H., et al. 1997. A CD4+ T-cell subset inhibits antigen-specific Tcell responses and prevents colitis. Nature. 389:737-742.

20. Chen, Y., Kuchroo, V.K., Inobe, J.-I., Hafler, D.A., and Weiner, H.L. 1994. Regulatory $\mathrm{T}$ cell clones induced by oral tolerance: suppression of autoimmune encephalomyelitis. Science. 265:1237-1240.

21. D'Orazio, T.J., and Niederkorn, J.Y. 1998. A novel role for TGF-beta and IL-10 in the induction of immune privilege. J. Immunol. 160:2089-2098.

22. Marth, T., Strober, W., and Kelsall, B.L. 1996. High dose oral tolerance in ovalbumin TCR-transgenic mice. J. Immunol. 157:2348-2357.

23. Yamamoto, H., Hirayama, M., Genyea, C., and Kaplan, J. 1994. TGF-beta mediates natural suppressor activity of IL-2-activated lymphocytes. $J$. Immunol. 152:3842-3847.

24. Inge, T.H., Hoover, S.K., Susskind, B.M., Barrett, S.K., and Bear, H.D 1992. Inhibition of tumor-specific cytotoxic T-lymphocyte responses by transforming growth factor beta 1. Cancer Res. 52:1386-1392.

25. Qin, L., Ding, Y., and Bromberg, J.S. 1996. Gene transfer of transform ing growth factor-beta 1 prolongs murine cardiac allograft survival by inhibiting cell-mediated immunity. Hum. Gene Ther. 7:1981-1988.

26. Hewitt, C.W., et al. 1997. Rat renal allograft tolerance is associated with local TGF-beta and absence of IL-2r expression within chimeric immunocytic foci. Transplant. Proc. 29:2183-2184.

27. Xu, X.Y., Honjo, K., Devore-Carter, D., and Bucy, R.P. 1997. Immunosuppression by inhibition of cellular adhesion mediated by leukocyte function-associated antigen-1/intercellular adhesion molecule- 1 in murine cardiac transplantation. Transplantation. 63:876-885.

28. Gorczynski, R.M., and Wojcik, D. 1994. A role for nonspecific (cyclosporin A) or specific (monoclonal antibodies to ICAM-1, LFA-1, and IL-10) immunomodulation in the prolongation of skin allografts after antigen-specific pretransplant immunization or transfusion. $J$. Immunol. 152:2011-2019.

29. Takeuchi, T., Lowry, R.P., and Konieczny, B. 1992. Heart allografts in murine systems. The differential activation of Th2-like effector cells in peripheral tolerance. Transplantation. 53:1281-1294.

30. Larsen, C.P., et al. 1996. Long-term acceptance of skin and cardiac allografts after blocking CD40 and CD28 pathways. Nature. 381:434-438.

31. Hall, B.M. 1991. Cells mediating allograft rejection. Transplantation. 51:1141-1151.

32. Perry, L.L., and Williams, I.R. 1995. Regulation of transplantation immunity in vivo by monoclonal antibodies recognizing host class II restric- tion elements. I. Genetics and specificity of anti-Ia immunotherapy in murine skin allograft recipients. J. Immunol. 134:2935-2941.

33. Loveland, B.E., and McKenzie, I.F.C. 1982. Delayed-type hypersensitivity and allograft rejection in the mouse: correlation of effector cell phenotype. Immunology. 46:313-320.

34. Sirak, J., Orosz, C.G., Wakely, E., and VanBuskirk, A.M. 1997. Alloreactive delayed-type hypersensitivity in graft recipients: complexity of responses and divergence from acute rejection. Transplantation. 63:1300-1307.

35. Hutchinson, I.V., Barber, W.H., and Morris, P.J. 1985. Specific suppression of allograft rejection by trinitrophenyl (TNP)-induced suppressor cells in recipients treated with TNP-haptenated donor alloantigens. $J$. Exp. Med. 162:1409-1420.

36. Qin, S., et al. 1993. "Infectious" transplantation tolerance. Science. 259:974-976.

37. Cobbold, S.P., Adams, E., Marshall, S.E., Davies, J.D., and Waldmann, H. 1996. Mechanisms of peripheral tolerance and suppression induced by monoclonal antibodies to CD4 and CD8. Immunol. Rev. 149:5-33.

38. Onodera, K., et al. 1996. Induction of "infectious" tolerance to MHCincompatible cardiac allografts in CD4 monoclonal antibody-treated sensitized rat recipients. J. Immunol. 157:1944-1950.

39. Niederkorn, J.Y., and Streilein, J.W. 1983. Alloantigens placed into the anterior chamber of the eye induce specific suppression of delayed-type hypersensitivity but normal cytotoxic T lymphocyte and helper T lymphocyte responses. J. Immunol. 131:2670-2674.

40. Streilein, J.W., Takeuchi, M., and Taylor, A.W. 1997. Immune privilege, T-cell tolerance, and tissue-restricted autoimmunity. Hum. Immunol. 52:138-143.

41. Hutchinson, I.V., and Morris, P.J. 1986. The role of major and minor histocompatibility antigens in active enhancement of rat kidney allograft survival by blood transfusion. Transplantation. 41:166-170.

42. Valujskikh, A., et al. 1998. T cells reactive to a single immunodominant self-restricted allopeptide induce skin graft rejection in mice. J. Clin. Invest. 101:1398-1407.

43. Verbanac, K.M., Carver, F.M., Haisch, C.E., and Thomas, J.M. 1994. A role for transforming growth factor-beta in the veto mechanism in transplant tolerance. Transplantation. 57:893-900.

44. Josien, R., et al. 1998. A critical role for transforming growth factor-beta in donor transfusion-induced allograft tolerance. J. Clin. Invest. 102:1920-1926.

45. Taga, K., and Giovanna, T. 1992. IL-10 inhibits human T cell proliferation and IL-2 production. J. Immunol. 148:1143-1148.

46. Qian, S., et al. 1994. Murine liver allograft transplantation: tolerance and donor cell chimerism. Hepatology. 19:916-924.

47. Starzl, T.E., Murase, N., Thomson, A., and Demetris, A.J. 1996. Liver transplants contribute to their own success. Nat. Med. 2:163-165.

48. Everson, G.T., et al. 1999. Early steroid withdrawal in liver transplantation is safe and beneficial. Liver Transpl. Surg. 5(Suppl. 1):S48-S57.

49. Fung, J.J. 1999. Toward tolerance: lessons learned from liver transplantation. Liver Transpl. Surg. 5(Suppl. 1):S90-S97.

50. Qian, S., et al. 1997. Apoptosis within spontaneously accepted mouse liver allografts: evidence for deletion of cytotoxic $\mathrm{T}$ cells and implications for tolerance induction. J. Immunol. 158:4654-4661.

51. Dahmen, U., et al. 1994. Split tolerance induced by orthotopic liver transplantation in mice. Transplantation. 58:1-8.

52. Olver, S., Goto, S., Chiba, S., Clouston, A., and Kelso, A. 1998. Persistence of donor-reactive CD4+ T cells in liver and spleen of rats tolerant to a liver allograft. Transplantation. 66:132-135.

53. Burlingham, W.J., et al. 2000. Tumor necrosis factor-alpha and tumor growth factor-beta 1 genotype: partial association with intragraft gene expression in two cases of long-term peripheral tolerance to a kidney transplant. Transplantation. 69:1527-1530.

54. Tran, H.M., et al. 1997. Distinct mechanisms for the induction and maintenance of allograft tolerance with CTLA4-Fc treatment. J. Immunol. 159:2232-2239.

55. Gudmundsdottir, H., and Turka, L.A. 1999. T cell costimulatory blockade: new therapies for transplant rejection. J. Am. Soc. Nephrol. 10:1356-1365.

56. Li, X.C., et al 1999. Induction of allograft tolerance in the absence of Fasmediated apoptosis. J. Immunol. 163:2500-2507.

57. Asiedu, C., et al. 1999. Immunoregulatory role of CD8alpha in the veto effect. Transplantation. 67:372-380.

58. Kabelitz, D. 1998. Apoptosis, graft rejection, and transplantation tolerance. Transplantation. 65:869-875

59. Jacobs, M.J.M., VanDenHoek, A.E.M., VanDePutte, L.B.A., and VanDenBerg, W.B. 1994. Anergy of antigen-specific T lymphocytes is a potent mechanism of intravenously induced tolerance. Immunology. 82:294-300.

60. Malvey, E.-N., Jenkins, M.K., and Mueller, D.L. 1998. Peripheral immune tolerance blocks clonal expansion but fails to prevent the differentiation of Th1 cells. J. Immunol. 161:2168-2177. 